\title{
A novel method for prenylquinone profiling in plant tissues by ultra-high pressure liquid chromatography-mass spectrometry
}

\author{
Jacopo Martinis ${ }^{1}$, Felix Kessler ${ }^{1}$ and Gaetan Glauser ${ }^{2 *}$
}

\begin{abstract}
Background: Prenylquinones are key compounds of the thylakoid membranes in chloroplasts. To understand the mechanisms involved in the response of plants to changing conditions such as high light intensity, the comprehensive analysis of these apolar lipids is an essential but challenging step. Conventional methods are based on liquid chromatography coupled to ultraviolet and fluorescence detection of a single or limited number of prenylquinones at a time. Here we present an original and rapid approach using ultra-high pressure liquid chromatography-atmospheric pressure chemical ionization-quadrupole time-of-flight mass spectrometry (UHPLCAPCI-QTOFMS) for the simultaneous profiling of eleven prenylquinones in plant tissues, including $\alpha$-tocopherol, phylloquinone, plastochromanol-8 and plastoquinone-9.

Results and discussion: Mass spectrometry and chromatography parameters were optimized using pure standards. Sample preparation time was kept to minimum and different extraction solvents were evaluated for yield, ability to maintain the redox state of prenylquinones, and compatibility with chromatography. In addition to precise absolute quantification of 5 prenyllipids for which standards were available, relative quantification of 6 other related compounds was possible thanks to the high identification power of QTOFMS. Prenylquinone levels were measured in leaves of Arabidopsis grown under normal and high light intensities. Quantitatively, the obtained results were consistent with those reported in various previous studies, demonstrating that this new method can profile the full range of prenylquinones in a very short time.

Conclusion: The new profiling method proves faster, more sensitive and can detect more prenylquinones than current methods based on measurements of selected compounds. It enables the extraction and analysis of twelve samples in only $1.5 \mathrm{~h}$ and may be applied to other plant species or cultivars.
\end{abstract}

Keywords: Prenylquinones, ultra-high pressure liquid chromatography, quadrupole-time-of-flight mass spectrometry, light stress, Arabidopsis thaliana

\section{Background}

The exposure to high light (HL) intensities is a normal event for plants growing under field conditions and represents a source of stress to the photosynthetic apparatus, both by direct photodamage and because of the formation of reactive oxygen species (ROS). Higher plants have evolved an array of mechanisms in order to dissipate the excess energy and protect themselves from

\footnotetext{
* Correspondence: gaetan.glauser@unine.ch

${ }^{2}$ Chemical Analytical Service of the Swiss Plant Science Web, University of Neuchâtel, 2000 Neuchâtel, Switzerland

Full list of author information is available at the end of the article
}

potential damage. In addition to state transition and changes in chloroplast ultrastructure, leaves acclimate to variations in light conditions through the accumulation of various antioxidants and the turnover of key photosystem components and reduced electron carriers. Prenylquinones, in particular, play a fundamental role in this process and include molecules with a broad action spectrum [1]. Plastoquinone-9 and ubiquinone-9, for example, are the two main lipophilic electron carriers in chloroplasts and mitochondria, respectively. In chloroplasts, $\alpha$-tocopherol (vitamin E) acts as the major antioxidant together with the reduced form of plastoquinone- 9 
and its derivative plastochromanol-8, protecting membranes from photoxidative stress [2-8]. Phylloquinone (Vitamin $\mathrm{K}_{1}$ ), on the other hand, is also a strong antioxidant but its main biological role is as one-electron carrier in the $A_{1}$ site of photosystem I. Moreover, it has been demonstrated that in cyanobacteria phylloquinone can be replaced by plastoquinone- 9 in the active site, but this does not occur in higher plants [9-11]. There is increasing evidence that prenylquinone metabolic pathways closely intersect [12] (Figure 1) and thus a suitable analytical technique enabling the detection of global and subtle changes in the profile of these thylakoid lipids is required. Most of the current methods for the analysis of prenylquinones are based on their extraction by an organic solvent followed by chromatographic analysis of selected molecules. However, the solvent choice may represent a major limiting factor for the extraction and chromatography efficiency. In addition to their variable extractive power related to the polarity of each molecule, some of the commonly used protic solvents may promote the spontaneous oxidation of the reduced forms of some lipids. The extraction protocol can also influence the stability and the redox state of the extracted molecules: in particular, reduced forms can be easily oxidized by atmospheric oxygen if samples are exposed to air or to high temperatures for a prolonged period of time. The separation and detection of the components of thylakoid membranes represents another key factor to obtain unambiguous lipid profiles. Recent methods are based on normal or reverse-phase high performance liquid chromatography (HPLC) coupled to ultraviolet and fluorescence detection [12-14]. These techniques require long running times (several dozens of minutes) to obtain sufficient resolution and the identification of lipid constituents relies on the availability of pure standards. Mass spectrometry, despite its high potential for the detection and identification of various apolar lipids [15], has been scarcely employed in combination with liquid chromatography for the analysis of prenyllipids in plants.

In this paper we propose a novel method for prenylquinone analysis using an optimized sample preparation procedure followed by ultra-high pressure liquid chromatography-atmospheric pressure chemical ionizationquadrupole time of flight mass spectrometry (UHPLCAPCI-QTOFMS). Compared to conventional HPLC, UHPLC uses sub-2 $\mu \mathrm{m}$ particle supports which allows for higher efficiency and optimal velocity [16,17]. Consequently, high throughput separations can be obtained by reducing column lengths and increased flow rates. The QTOF mass spectrometer is particularly well adapted to coupling with UHPLC thanks to its rapid scanning rate [18]. Moreover its high mass accuracy gives access to the determination of molecular formula, an essential feature for reliable compound identification. Different solvents were tested for their extraction yield, their ability to maintain the redox state of molecules as well as their compatibility with reverse-phase UHPLC as injection solvent. Chromatography and mass spectrometry parameters were optimized for speed, selectivity and sensitivity. The applicability of the developed method was illustrated with the simultaneous quantification of several prenylquinones in Arabidopsis thaliana grown under normal and high light conditions.

\section{Results and Discussion}

\section{Optimization of MS conditions}

While there have been several publications on the LCMS analysis of tocopherols [19,20] and vitamin K homologues [21,22] as they are essential vitamins for human metabolism, we are not aware of any report for plastoquinone-9 (PQ-9) and plastochromanol-8 (PC-8). Thus different QTOFMS parameters were evaluated to obtain maximal sensitivity for these latter molecules together with $\alpha$-tocopherol $(\alpha-\mathrm{T})$ and phylloquinone (K). Electrospray and APCI were compared in both positive and negative ionization modes using standard solutions at 1 $\mu \mathrm{g} / \mathrm{mL}$. For PQ-9, the oxidized form (PQ) was used as standard. APCI was found largely superior to electrospray for all four compounds. K, PQ and PC-8 gave similar responses in positive and negative APCI but $\alpha-\mathrm{T}$ was much better ionized in negative mode (ca. 8-fold), which was thus selected for further experiments. We then tested the effect of the probe temperature on the ionization efficiency. While $\alpha-\mathrm{T}$ and $\mathrm{K}$ gave a higher signal at lower temperatures $\left(350-500^{\circ} \mathrm{C}\right), \mathrm{PC}-8$ and PQ were best ionized at higher temperatures $\left(475-600^{\circ} \mathrm{C}\right)$. A temperature of $475^{\circ} \mathrm{C}$ was selected as the best compromise for the detection of the four compounds. The source cone voltage was varied from 15 to $50 \mathrm{~V}$ and the highest response was obtained at $40 \mathrm{~V}$. Other source parameters such as corona current $(18 \mu \mathrm{A})$, source temperature $\left(120^{\circ} \mathrm{C}\right)$ and desolvation gas flow $(800 \mathrm{~L} / \mathrm{hr})$ had a less important effect on the MS signal. The influence of the mobile phase composition on the ionization efficiency was evaluated. Methanol $(\mathrm{MeOH})$, acetonitrile (ACN) and tetrahydrofuran (THF) were compared and $\mathrm{MeOH}$ gave the best signal for all compounds. For $\alpha-\mathrm{T}$ and $\mathrm{K}$, the intensity of MS responses in $\mathrm{MeOH}$ was 2-5 fold higher than in ACN or THF. For PQ and PC-8 it was 10-40 fold higher. $\mathrm{MeOH}$ was thus selected as the organic solvent of choice for further LC method development.

\section{Optimization of LC conditions}

Prenyllipids are traditionally separated by isocratic normal-phase chromatography using diol columns and very apolar solvents or by reverse-phase (RP) chromatography using octadecyl (C18) columns and mixtures of 


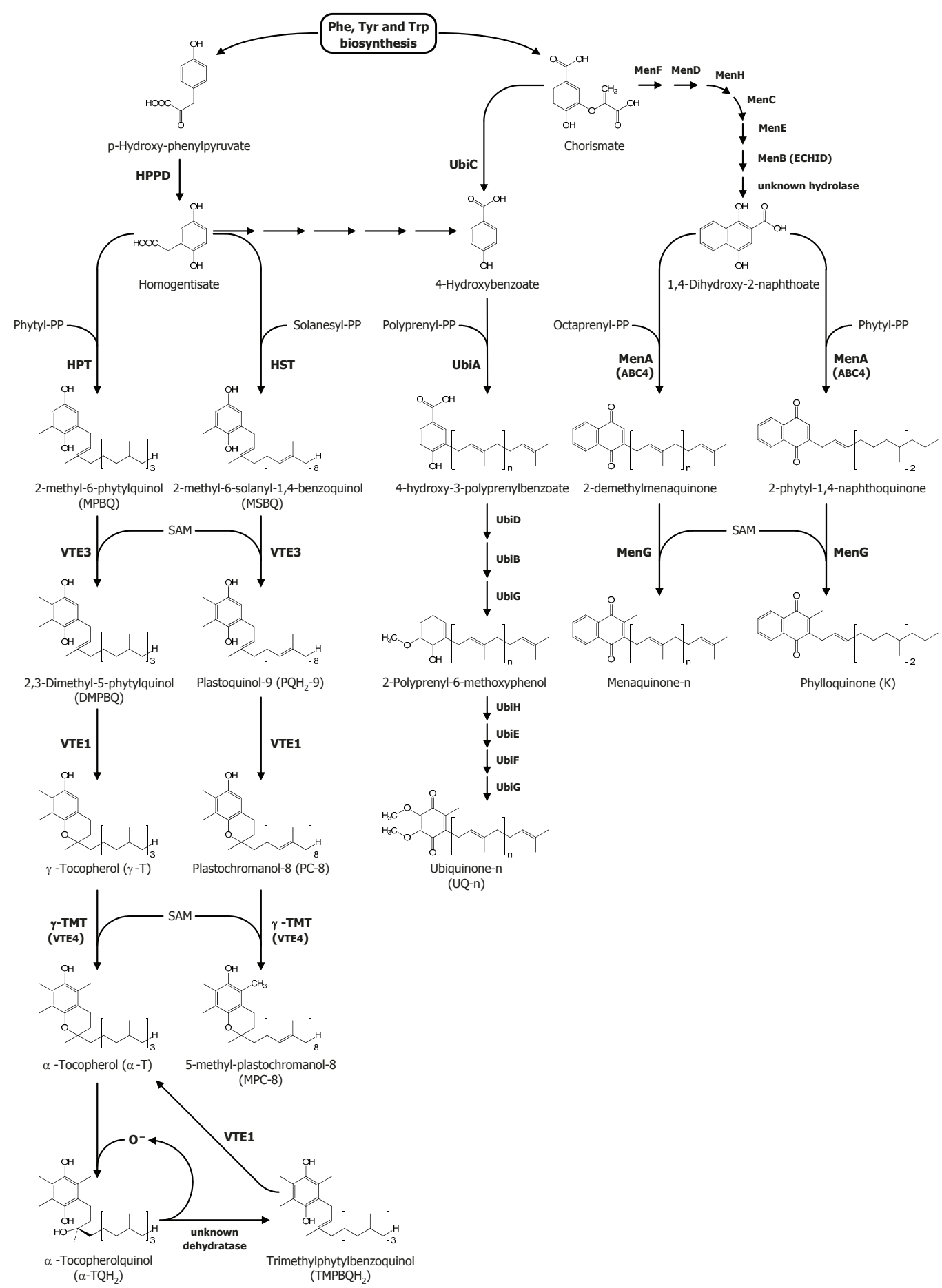

Figure 1 Biosynthetic pathways of tocopherol, plastoquinol, plastochromanol, ubiquinone, and phylloquinone in Arabidopsis.

$\mathrm{MeOH} /$ Ethanol or ACN/Ethanol as mobile phases. In RP mode, small amounts of hexane are sometimes used to accelerate the elution of PQ and PC-8 which are more hydrophobic than $\alpha-\mathrm{T}$ and $\mathrm{K}$. Run times are quite long (typically 20-40 min) because complete resolution of the peaks is needed for accurate quantification by ultraviolet and fluorescence detection. Using MS as a detector however, resolution becomes less important and chromatographic separations can often be shortened. In this study, UHPLC coupled to QTOFMS detection was evaluated for the high throughput analysis of prenyllipids. A $50 \times 2.1 \mathrm{~mm} \mathrm{C18}$ column filled with 
$1.7 \mu \mathrm{m}$ particles was selected for the separation. We first tested an isocratic $100 \% \mathrm{MeOH}$ mobile phase at $25^{\circ} \mathrm{C}$. Adequate retention factors $(\mathrm{k})$ were obtained for $\alpha-\mathrm{T}(\mathrm{k}$ $=2.3)$ and $\mathrm{K}(\mathrm{k}=3.9)$, but $\mathrm{PC}-8(\mathrm{k}=11.7)$ and PQ $(\mathrm{k}=$ 17) were too strongly retained leading to broad peaks and a decrease in sensitivity (Figure 2A). Since the use of $\mathrm{MeOH}$ as organic solvent was a required condition for MS sensitivity, we could not replace it by a solvent of higher eluting power. An alternative option to decrease the retention of $\mathrm{PC}-8$ and $\mathrm{PQ}$ was to increase the temperature of the column. At $60^{\circ} \mathrm{C}$, the retention factors for PC-8 and PQ were reduced to appropriate values, respectively 4.5 and 6.3. Meanwhile, the retention for $\alpha-T$ and $K$ became slightly too weak with $k$ values of 1.1 and 1.9 respectively (Figure $2 \mathrm{~B}$ ). To overcome this issue, a 1.5 min gradient from $\mathrm{H}_{2} \mathrm{O} / \mathrm{MeOH}$ $(10: 90, \mathrm{v} / \mathrm{v})$ to $100 \% \mathrm{MeOH}$ was implemented at the beginning of the run followed by a hold at $100 \% \mathrm{MeOH}$ for $2.5 \mathrm{~min}$. Using this optimized separation, all compounds of interest eluted between 0.9 and $2.5 \mathrm{~min}$ and gave symmetrical and sharp peak shapes (Figure $2 \mathrm{C}$ ). We verified that no thermal degradation occurred at $60^{\circ}$ C. Although prenyllipids are neutral molecules and their retention times are not likely to be affected by a possible

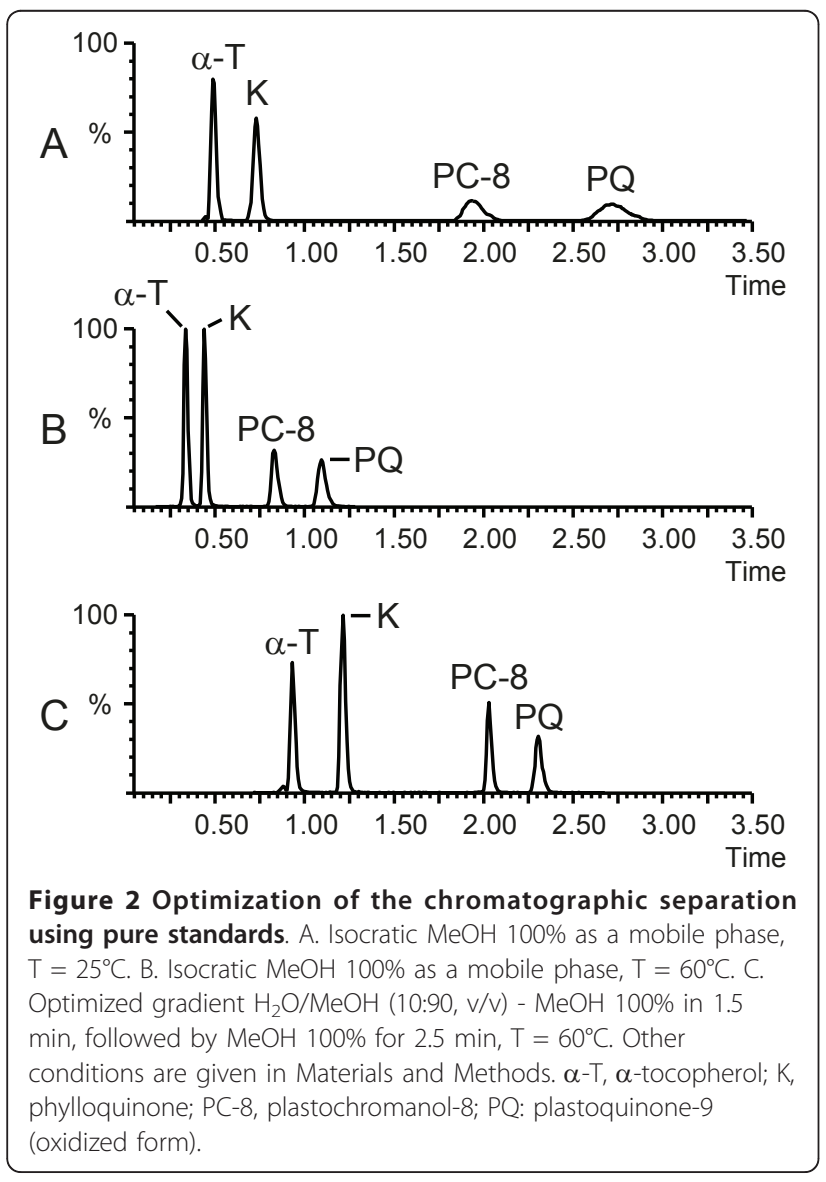

change in $\mathrm{pH}$, we also tested the impact of mobile phase additives such as formic acid in terms of retention and ionization efficiency. As expected, no change in retention times was observed. On the contrary, the ionization efficiency was reduced by one order of magnitude for $\mathrm{K}$ and PQ, and more than two orders of magnitude for $\alpha$ $\mathrm{T}$ and $\mathrm{PC}-8$. This is not surprising since strong gas phase acids suppress proton transfers in negative APCI. It was found that even the injection of an acidic solution of standards could slightly reduce the ionization efficiency of $\alpha-T$ even if mobiles phases without additive were used. For this reason it is recommended to remove any trace of formic acid in the system, including the injector wash lines (if partial loop mode is employed), before prenyllipids are analyzed. Acids are also frequently used for lipid extractions in order to inhibit enzymatic hydrolysis. In the case of prenyllipids, this should be avoided.

\section{Detection of prenylquinones in plant samples}

To evaluate the performance of the developed UHPLCAPCI-QTOFMS method on biological samples, we extracted Arabidopsis plants grown under normal and high light conditions in a mixture of chloroform/methanol $\left(\mathrm{CHCl}_{3} / \mathrm{MeOH}, 30: 70, \mathrm{v} / \mathrm{v}\right)$. $\alpha-\mathrm{T}, \mathrm{K}, \mathrm{PC}-8$, and the oxidized $(\mathrm{PQ})$ and reduced $\left(\mathrm{PQH}_{2}\right)$ forms of $\mathrm{PQ}-9$ were easily detected. The presence of $\mathrm{PQH}_{2}$ was confirmed by reducing the PQ standard with sodium borohydride and analyzing the resulting mixture. For these 5 molecules, positive identification was achieved based on the comparison of retention times and accurate masses with pure available standards. In addition, the QTOFMS allowed for the tentative identification of six other prenyllipids for which no standard was available, namely $\gamma$ tocopherol $(\gamma-\mathrm{T}), \alpha$-tocopherol quinone $(\alpha-\mathrm{TQ})$, hydroxyplastochromanol (PC-OH), hydroxyplastoquinone $(\mathrm{PQ}-\mathrm{OH})$ and the oxidized (UQ) and reduced $\left(\mathrm{UQH}_{2}\right)$ forms of ubiquinone-9 (UQ-9). UHPLC-APCI-QTOFMS data acquired in negative and positive APCI for the identified prenyllipids are presented in Table 1.

\section{Optimization of sample preparation}

The above used extraction solvent $\left(\mathrm{CHCl}_{3} / \mathrm{MeOH}, 30: 70\right.$ $\mathrm{v} / \mathrm{v})$ seemed to efficiently extract prenyllipids. However it had a serious drawback: $\mathrm{CHCl}_{3}$ is chemically poorly compatible with the UHPLC system used which can accommodate all common solvents except chlorinated solvents. We therefore evaluated different extraction solvents based on the three following criteria: extraction yield for the prenylquinones, ability to maintain the redox state of $\mathrm{PQ} / \mathrm{PQH}_{2}$ and $\mathrm{UQ} / \mathrm{UQH}_{2}$, and compatibility with UHPLC in terms of peak broadening and chemical resistance. A preliminary condition was to avoid an evaporation-redissolution step which would 
Table 1 Prenylquinones identified from UHPLC-APCI-QTOFMS data acquired in negative and positive ionization modes

\begin{tabular}{cccccccccc}
\hline No & $\mathbf{R T}(\mathbf{m i n})$ & $\mathbf{A P C l}-$ & $\mathbf{m} / \mathbf{z}(-)$ & $\mathbf{A P C l}+$ & $\mathbf{m} / \mathbf{z}(+)$ & formula & MS/MS (-) & Identification \\
\hline 1 & 0.76 & $(\mathrm{M})^{-}$ & 446.3763 & $-^{a}$ & $-^{a}$ & $\mathrm{C}_{29} \mathrm{H}_{50} \mathrm{O}_{3}$ & $177.0919,163.0764$ & $\alpha$-tocopherol-quinone \\
\hline 2 & 0.92 & $(\mathrm{M}-\mathrm{H})^{-}$ & 415.3575 & $-^{-a}$ & $-^{a}$ & $\mathrm{C}_{28} \mathrm{H}_{48} \mathrm{O}_{2}$ & $149.0605,121.0655$ & $\gamma$-tocopherol \\
\hline 3 & 1.00 & $(\mathrm{M}-\mathrm{H})^{-}$ & 429.3734 & $(\mathrm{M}+\mathrm{H})^{+}$ & 431.3877 & $\mathrm{C}_{29} \mathrm{H}_{50} \mathrm{O}_{2}$ & $163.0760,135.0812$ & $\alpha$-tocopherol \\
\hline 4 & 1.28 & $(\mathrm{M})^{-}$ & 450.3504 & $(\mathrm{M}+\mathrm{H})^{+}$ & 451.3572 & $\mathrm{C}_{31} \mathrm{H}_{46} \mathrm{O}_{2}$ & $210.0680,185.0607$ & phylloquinone \\
\hline 5 & 1.52 & $\left(\mathrm{M}-\mathrm{H}^{-}\right.$ & 765.6183 & $-^{-}$ & $-^{a}$ & $\mathrm{C}_{53} \mathrm{H}_{81} \mathrm{O}_{3}$ & $750.5956,149.0608$ & hydroxyplastochromanol \\
\hline 6 & 1.71 & $(\mathrm{M})^{-}$ & 764.6102 & $-^{-a}$ & $-^{a}$ & $\mathrm{C}_{53} \mathrm{H}_{80} \mathrm{O}_{3}$ & 149.0604 & hydroxyplastoquinone \\
\hline 7 & 1.72 & $(\mathrm{M}-2 \mathrm{H})^{-}$ & 748.6158 & $(\mathrm{M}+\mathrm{H})^{+}$ & 751.6385 & $\mathrm{C}_{53} \mathrm{H}_{82} \mathrm{O}_{2}$ & $188.0836,149.0605$ & plastoquinol \\
\hline 8 & 1.89 & $(\mathrm{M}-2 \mathrm{H})^{-}$ & 794.6214 & $(\mathrm{M}+\mathrm{H})^{+}$ & 797.6448 & $\mathrm{C}_{54} \mathrm{H}_{84} \mathrm{O}_{4}$ & $779.5970,219.0661$ & ubiquinol \\
\hline 9 & 2.12 & $(\mathrm{M}-\mathrm{H})^{-}$ & 749.6227 & $(\mathrm{M}+\mathrm{H})^{+}$ & 751.6395 & $\mathrm{C}_{53} \mathrm{H}_{82} \mathrm{O}_{2}$ & $734.6009,149.0608$ & plastochromanol-8 \\
\hline 10 & 2.16 & $(\mathrm{M})^{-}$ & 794.6212 & $(\mathrm{M}+\mathrm{H})^{+}$ & 795.6300 & $\mathrm{C}_{54} \mathrm{H}_{82} \mathrm{O}_{4}$ & $779.5974,219.0655$ & ubiquinone \\
\hline 11 & 2.43 & $(\mathrm{M})^{-}$ & 748.6152 & $(\mathrm{M}+\mathrm{H})^{+}$ & 749.6232 & $\mathrm{C}_{53} \mathrm{H}_{80} \mathrm{O}_{2}$ & $188.0841,149.0606$ & plastoquinone \\
\hline
\end{tabular}

a, not detected. RT, retention time. Known chemical structures are available in Figure 1.

lengthen the sample preparation and possibly alter PQ/ $\mathrm{PQH}_{2}$ and $\mathrm{UQ} / \mathrm{UQH}_{2}$ redox ratios. Plants exposed to $\mathrm{HL}$ intensity $\left(500 \mu \mathrm{E} \cdot \mathrm{m}^{-2} \cdot \mathrm{s}^{-1}\right)$ were pooled and lipids were extracted using $\mathrm{MeOH}$, isopropanol (IPA), ethylacetate (EtAC), THF and $\mathrm{CHCl}_{3} / \mathrm{MeOH}(30: 70$, v/v) as control and further analyzed by UHPLC-APCIQTOFMS $(\mathrm{n}=4)$. A principal component analysis (PCA) was performed on the obtained data. Interestingly, the five solvents clustered separately (Figure 3) and showed a significant selectivity for different molecules. In general, polar lipids including $\alpha-T Q$ were extracted more efficiently using $\mathrm{MeOH}$, while more apolar solvents showed a higher extractive power for apolar prenyllipids (e.g. PQ and UQ). For all prenylquinones, the highest extraction yield was obtained with $\mathrm{CHCl}_{3}$ / $\mathrm{MeOH}(30: 70, \mathrm{v} / \mathrm{v})$ and THF, while it was lower for EtAc and IPA (Figure 4A-F). MeOH represented a separate case because of its higher polarity, resulting in poor ability to extract PQ-9 and PC-8. Therefore $\mathrm{MeOH}$ is not a suitable solvent for PQ-9 and PC-8 analysis (Figure $4 \mathrm{D}$ and $4 \mathrm{E})$.

Spontaneous variations in $\mathrm{PQ} / \mathrm{PQH}_{2}$ ratio under standard working conditions were determined in the five solvents using a purified PQ standard. No spontaneous reduction was detected (data not shown). Moreover, when spiking PQ in leaf extracts, no increase in $\mathrm{PQH}_{2}$ was observed, suggesting that the reducing agents present in plant tissues are not concentrated enough to promote reduction. However, when ascorbic acid (30 $\mathrm{mM}$ ) or butyl hydroxytoluene (BHT, $0.05 \%$ ) were added to $\mathrm{PQ}$ solutions prepared in the different solvents, a slight reduction of PQ was observed (about 3\% and 1\% of the original amount of PQ using ascorbic acid or $\mathrm{BHT}$, respectively). This phenomenon has previously been observed [13]. While anti-oxidant agents are often added to extraction experiments to prevent lipid peroxidation, this procedure does not appear useful for the determination of the redox state of PQ since reduction of the oxidized form may occur. When the PQ standard was chemically reduced with sodium borohydride and then exposed to air at room temperature, an increase of the oxidized form was detected for all solvents starting from 5-7 h after reduction. As a consequence, for the solvent to be effective in maintaining the original $\mathrm{PQ} / \mathrm{PQH}_{2}$ ratio, it needs to oxidize the $\mathrm{PQH}_{2}$ present in plant tissues as little as possible. Using $\mathrm{CHCl}_{3} / \mathrm{MeOH}(30: 70, \mathrm{v} / \mathrm{v})$, IPA and THF, a $\mathrm{PQH}_{2} /$ $\mathrm{PQ}_{\text {Tот }}$ ratio $\geq 0.5$ was found in plants exposed to $\mathrm{HL}$ conditions, while EtAc led to a $\mathrm{PQH}_{2} / \mathrm{PQ}_{\mathrm{TOT}}$ ratio of only 0.3 (Figure 4D). This may be due to lower extraction yield for $\mathrm{PQH}_{2}$ in EtAc rather than oxidation of $\mathrm{PQH}_{2}$ into $\mathrm{PQ}$. Interestingly, when plants grown under normal light intensity $\left(150 \mu \mathrm{E} \cdot \mathrm{m}^{-2} \cdot \mathrm{s}^{-1}\right)$ were extracted in IPA, a complete oxidation of $\mathrm{PQH}_{2}$ was observed, while this did not happen in samples extracted by $\mathrm{CHCl}_{3} /$ $\mathrm{MeOH}(30: 70, \mathrm{v} / \mathrm{v})$ and THF (data not shown). This may be explained by the higher amount of antioxidants (tocopherols or other lipid-soluble molecules) naturally present in thylakoid membranes under HL conditions, which could have protected $\mathrm{PQH}_{2}$ from spontaneous oxidation in IPA. A similar profile was observed for UQ-9 (Figure 4F).

The influence of the extraction solvents on the chromatographic performance was also evaluated. Indeed, it is well-known that injection solvents of higher elution strength than the initial mobile phase can lead to peak distortion and broadening, in particular for early eluting peaks. No significant peak broadening was observed for $\mathrm{MeOH}, \mathrm{CHCl}_{3} / \mathrm{MeOH}(30: 70, \mathrm{v} / \mathrm{v})$ and IPA (Figure 5AC). Using THF, which is a stronger solvent, $\gamma-T, \alpha-T$ and $\mathrm{K}$ exhibited moderate peak broadening (Figure 5D). Not surprisingly, EtAc was the most problematic 


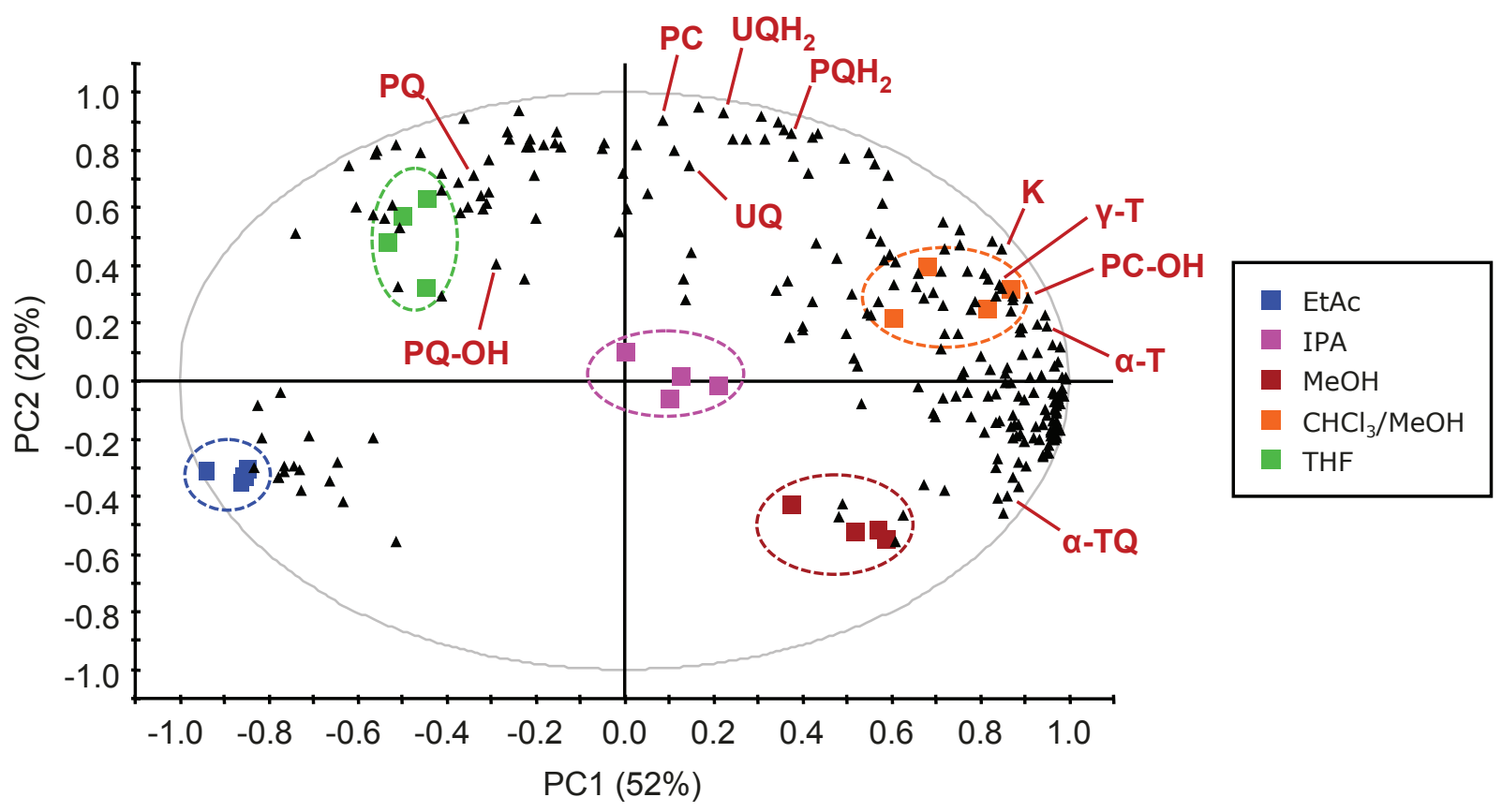

Figure 3 Bi-plot derived from a principal component analysis (PCA) showing the selectivity of the extraction solvent. Plants exposed to high light were pooled and extracted using the indicated solvents. Colored squares correspond to the observations $(n=4$ for each solvent) and black triangles represent the variables. PC1 and PC2 are first and second principal components, respectively, with their percentage of explained variance. The identified prenyllipids are indicated (confront with Table 1). For abbreviations, see text.

solvent: strong peak distortion was observed for $\gamma-\mathrm{T}, \alpha-$ $\mathrm{T}$ and $\mathrm{K}$ and even $\mathrm{PQH}_{2}$ was affected (Figure $5 \mathrm{E}$ ). We then diminished the injection volume from 5 to $2.5 \mu \mathrm{L}$, which substantially reduced peak broadening for THF but only slightly for EtAc (data not shown). In conclusion, $2.5 \mu \mathrm{L}$ injections of solvents equally or more polar than THF could be used without negative effect on the chromatographic performance under the used conditions.

Overall, THF represented a good alternative to $\mathrm{CHCl}_{3} / \mathrm{MeOH}(30: 70, \mathrm{v} / \mathrm{v})$ since it provided high and reproducible extraction yields, best maintained the redox state of PQ-9 and UQ-9, and was chromatographically compatible. It was thus selected as the solvent of choice for the extraction of biological samples. To determine whether the volume of extraction solvent (500 $\mu \mathrm{L}$ THF for $100 \mathrm{mg}$ of fresh leaf material) was sufficient for extracting most of the prenylquinones, we extracted leaves exposed to HL in $1500 \mu \mathrm{L}$ or $500 \mu \mathrm{L}$ (for the latter, the extract was further diluted three times) and extraction yields were compared $(n=3)$. No significant difference was found, confirming that a volume of $500 \mu \mathrm{L}$ is sufficient for the extraction of 100 $\mathrm{mg}$ of fresh leaves. We noticed that the oxidation of $\mathrm{PQH}_{2}$ became significant about 2 and 5 hours after extraction for plants grown under normal and high light conditions respectively. For this reason, we took good care to always prepare and analyze samples within a maximum period of 1.5 hours. Given the speed of both extraction and analysis, 12 samples can be processed during that period of time. By performing sample preparation and analyses in parallel, a throughput of 100 samples in $8 \mathrm{~h}$ could be potentially achieved.

\section{Extraction recovery, matrix effects and limits of quantification}

To evaluate sample preparation recovery, two experiments were carried out: first pure standards of $\alpha-\mathrm{T}, \mathrm{K}$, $\mathrm{PC}$ and PQ were submitted to the extraction procedure. Recovery greater than $95 \%$ was obtained for all molecules. To determine if the plant matrix had an impact on the recovery, decyl-plastoquinone was used as nonendogenous structural analogue and spiked before and after extraction of plant samples at identical concentration. Again a recovery greater than $95 \%$ was obtained.

APCI is usually less prone to matrix effects than electrospray because ionization occurs in the gas phase. We nevertheless checked if prenyllipids were subjected to suppression or enhancement effects from the Arabidopsis extract. Since these molecules are endogenous in Arabidopsis, a THF extract was prepared and an aliquot was spiked with standard solutions of each prenyllipid. The control extract (C), the standard solutions (SS) and the spiked extract (SE) were injected and the obtained 

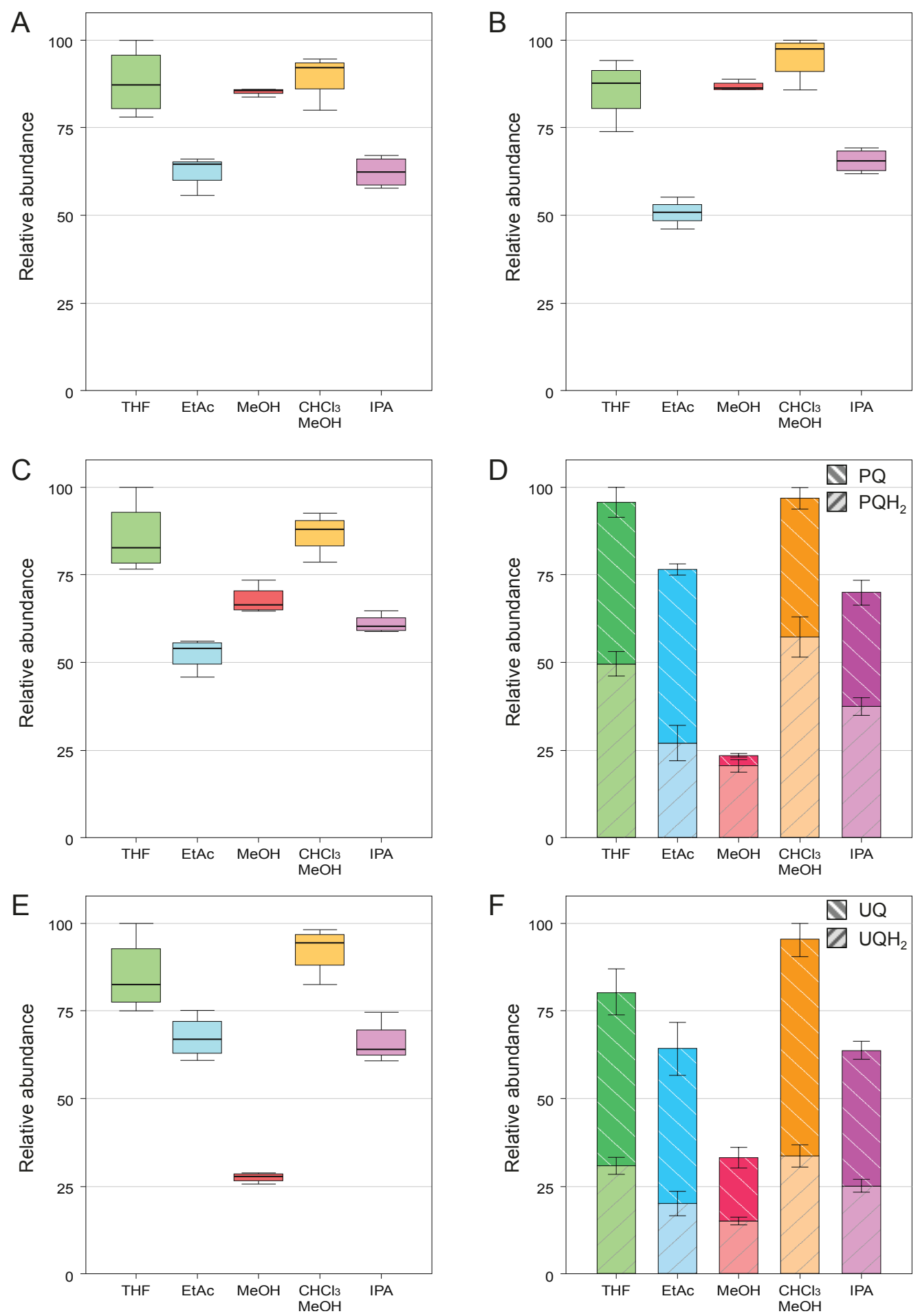

Figure 4 Comparison of the extraction yields for prenylquinones with the five selected solvents. A. $\gamma$-tocopherol. B. $\alpha$-tocopherol. C. Phylloquinone. D. Plastoquinone-9, oxidized (PQ) and reduced $\left(\mathrm{PQH}_{2}\right)$ forms. E. Plastochromanol-8. F. Ubiquinone-9, oxidized (UQ) and reduced $\left(\mathrm{UQH}_{2}\right)$ forms. Plants exposed to high light were pooled and lipids were extracted using the indicated solvents. Data are from 4 replicates $( \pm \mathrm{SD})$. For PQ-9 (D) and UQ-9 (F) the ability to maintain the redox state was also evaluated. 


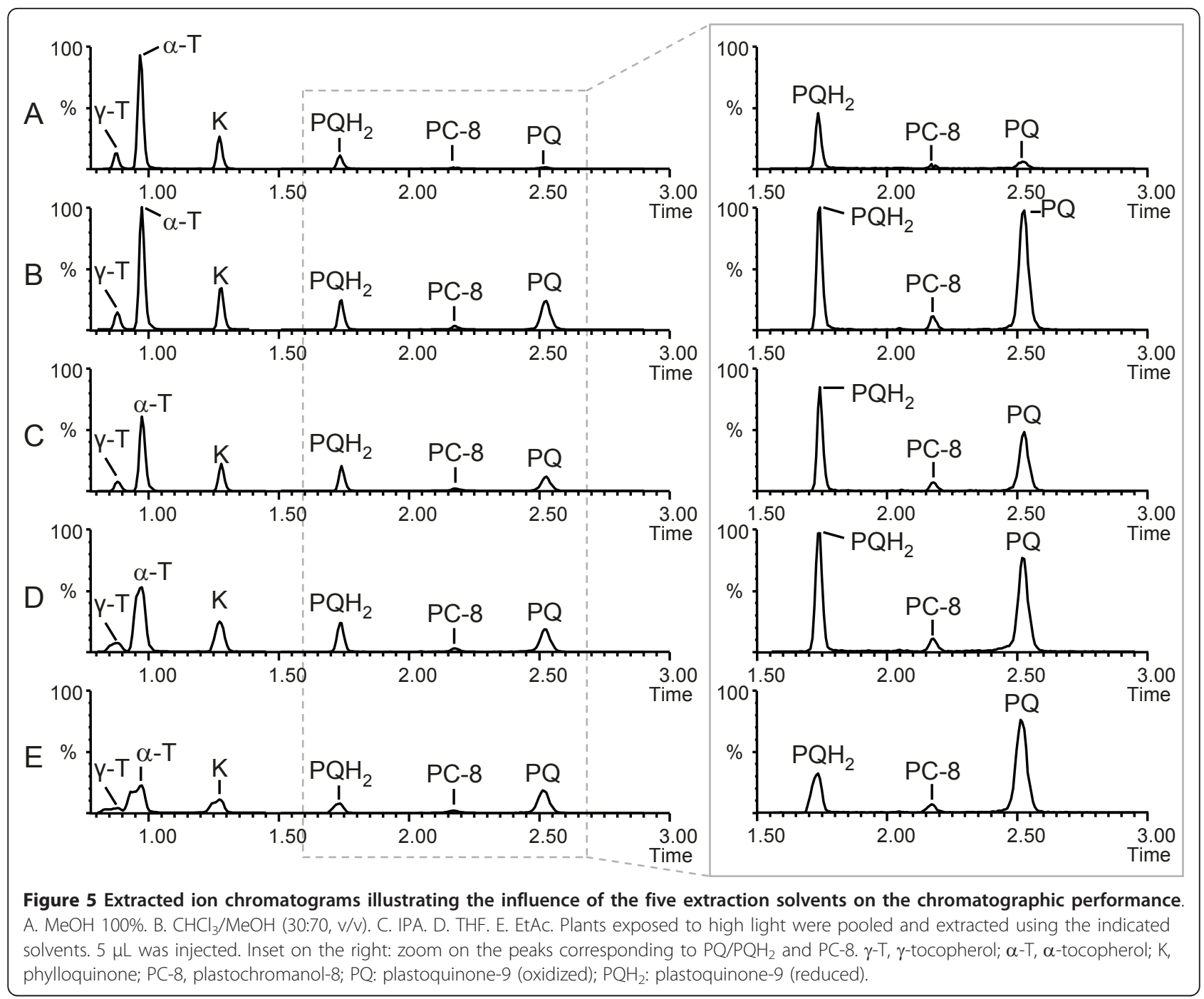

area compared. For all the ions, the area of $\mathrm{C}+\mathrm{SS}$ were equivalent to those of SE with a variation inferior to $5 \%$. In other words, no significant matrix effect was observed for the analyzed compounds.

The quantification of prenyllipids was based on internal standard calibration. Decyl-plastoquinone was found suitable as an internal standard: it was structurally close to the studied prenylquinones, was readily detected in negative APCI with high selectivity $\left(\mathrm{M}^{-}\right.$ion at $\mathrm{m} / z$ 276.2087), eluted within the chromatographic gradient (retention time $0.44 \mathrm{~min}$ ), and no matrix effect was observed. For $\alpha-T, P Q$ and PC-8, five calibrations points were used $(0.05,0.2,1.0,2.0$, and $10.0 \mu \mathrm{g} / \mathrm{mL}$ for $\alpha-\mathrm{T}$; $0.05,0.2,1.0,2.0$, and $5.0 \mu \mathrm{g} / \mathrm{mL}$ for PQ-9 and PC-8). When PQ was completely reduced using sodium borohydride, the peak corresponding to $\mathrm{PQH}_{2}$ had an identical area to that of $P Q$. As a result, $\mathrm{PQH}_{2}$ could be quantified based on PQ calibration curve. For K, whose concentration does not significantly change after HL treatment, only four calibration points were used $(0.1$, $0.25,1.0$, and $2.5 \mu \mathrm{g} / \mathrm{mL}$ ). For all compounds the response was linear over the range of the chosen concentrations with coefficients of determination $>0.99$. A signal-to-noise ratio $(\mathrm{s} / \mathrm{n})$ of 10 was defined as limit of quantification (LOQ). For $\alpha-\mathrm{T}, \mathrm{K}$ and PC-8, the LOQ was $20 \mathrm{ng} / \mathrm{mL}$. For PQ-9, an LOQ of $10 \mathrm{ng} / \mathrm{mL}$ was attained. The other identified prenyllipids (see Table 1) for which no pure standard was available were relatively quantified.

\section{Effect of high light on prenylquinone profile}

$\mathrm{HL}$ exposure induces biochemical and physiological changes in plants. In order to provoke a strong effect on prenyllipid content, 4 to 5-week-old Arabidopsis thaliana (Columbia-0) plants were exposed to continuous HL intensity for a prolonged period of time (7 days) and their prenyllipid profiles were compared to those obtained from plants grown under normal light. Under 

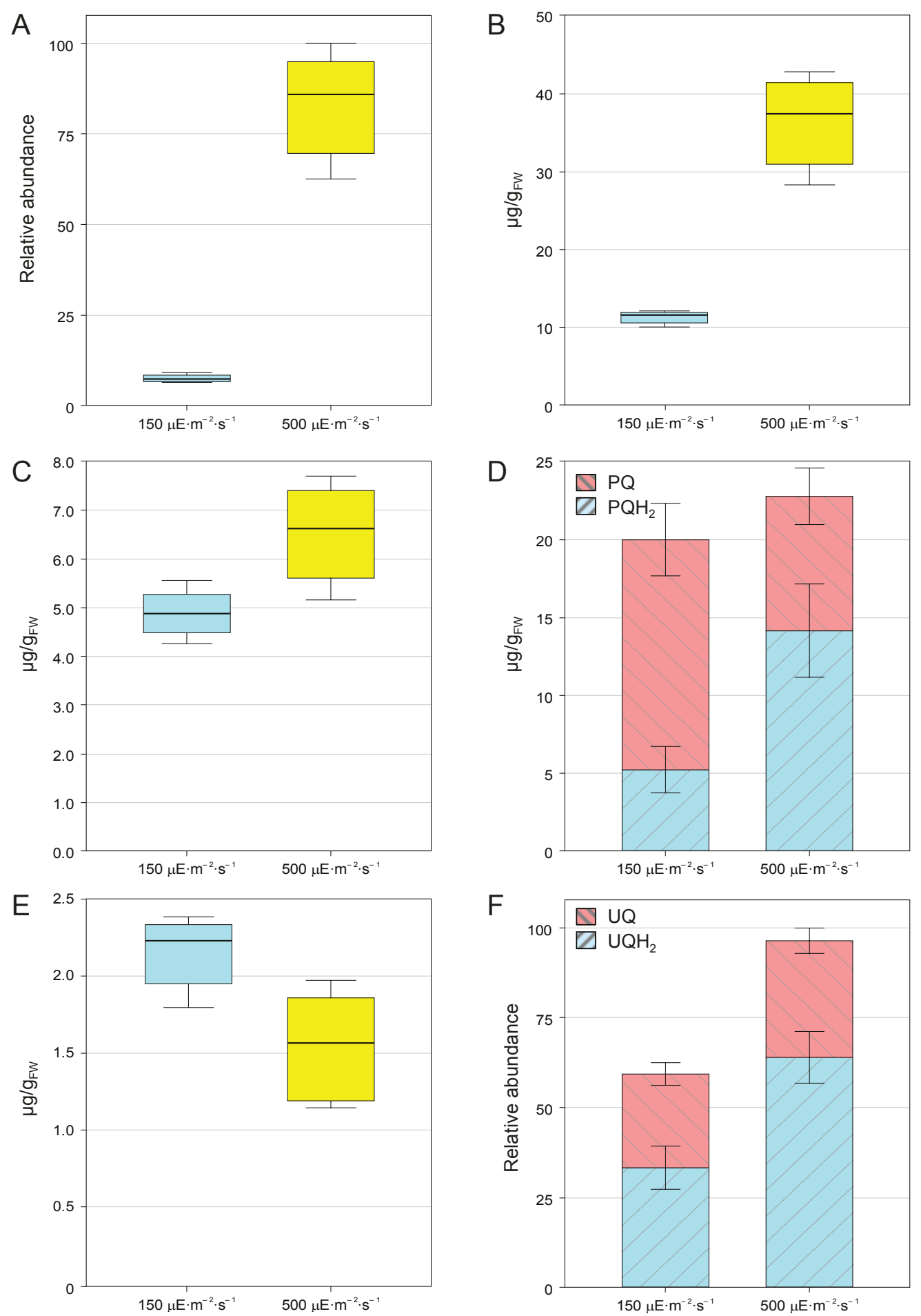

Figure 6 Effect of $\mathrm{HL}$ exposure on the total leaf content of prenylquinones. A. $\gamma$-tocopherol. B. $\alpha$-tocopherol. C. Phylloquinone. D. Plastoquinone-9, oxidized ( $\mathrm{PQ}$ ) and reduced $\left(\mathrm{PQH}_{2}\right)$ forms. E. Plastochromanol-8. F. Ubiquinone-9, oxidized (UQ) and reduced $\left(\mathrm{UQH}_{2}\right)$ forms. Plants exposed to normal $\left(150 \mu \mathrm{E} \times \mathrm{m}^{-2} \times \mathrm{s}^{-1}\right)$ or high $\left(500 \mu \mathrm{E} \times \mathrm{m}^{-2} \times \mathrm{s}^{-1}\right)$ light intensities were extracted using THF. Data are from 4 biological replicates $( \pm$ SD). For PQ-9 (D) and UQ-9 (F) the redox state of the total leaf pool was taken into account. 
HL conditions $\gamma$ - and $\alpha$-tocopherol levels increased 8fold and 3.5-fold respectively (Figure 6A and 6B). The synthesis of tocopherols and their accumulation in thylakoid membranes and plastoglobules is a well-known phenomenon which has been demonstrated to occur in response to oxidative stress [4,5]. After exposure to HL conditions plants produce more $\alpha$-tocopherol, which mitigates photosystem II photoinactivation and protects thylakoids from photooxidative damage under chilling conditions $[6,23]$. No significant variation in phylloquinone levels was observed under HL (Figure 6C). As a matter of fact, even though its absence in plants lacking key enzymes in vitamin $\mathrm{K}$ biosynthetic pathway affects photosystem I activity [9], no direct evidence of its accumulation under HL has been reported [4]. In the future, we will apply this new method to profile simultaneously several tocochromanols/prenylquinones and assess the roles of each of them under various conditions.

Concerning plastoquinone-9, 4-week-old $A$. thaliana plants grown under normal light conditions showed a $\mathrm{PQH}_{2} / \mathrm{PQ}_{\mathrm{TOT}}$ ratio of about 0.25 , in agreement with a previous study by Szymanska et al. [24] that used plants of similar age and a different method for prenyllipids extraction. After continuous HL exposure, plastoquinone-9 total content (oxidized + reduced) did not seem to be significantly altered. Yet, the redox state of the electron acceptor pool changed, with a significant accumulation of the reduced form $\left(\mathrm{PQH}_{2}\right)$, leading to a $\mathrm{PQH}_{2} / \mathrm{PQ}_{\mathrm{TOT}}$ ratio of 0.6 (Figure $6 \mathrm{D}$ ). This result suggests that the accumulation of $\mathrm{PQH}_{2}$ under $\mathrm{HL}$ may not be due to the de novo synthesis of the latter but on the reduction of the already available PQ pool. While these findings are distinct from the increase in $\mathrm{PQH}_{2}$ synthesis reported by Szymanska et al. [24], this discrepancy may well be attributed to the different growth and light conditions or to the different reference units employed ( $\mu \mathrm{g} / \mathrm{g}$ fresh weight versus $\mu \mathrm{g} / \mathrm{mg}$ chlorophyll).

Among the other prenyllipids identified, plastochromanol-8 levels did not significantly change when plants were exposed to HL (Figure 6E), while total ubiquinone9 content increased about 1.5-fold. Moreover, the $\mathrm{UQH} 2 / \mathrm{UQ}_{\mathrm{TO}}$ ratio increased in response to the change in light conditions (Figure 6F), as previously observed by Yoshida et al. [14].

\section{Conclusion}

The presented method introduces for the first time the use of UHPLC-APCI-QTOFMS for simultaneously profiling several prenylquinones in plants. It proves to be fast, reliable, very selective and sensitive for the analyzed molecules, and consume less solvent than conventional methods. By combining it with simple and rapid sample preparation, a single plant can be extracted and analyzed in less than $15 \mathrm{~min}$ and twelve samples can be processed in $90 \mathrm{~min}$. Moreover it allows for the detection and tentative identification of molecules for which no pure standard is available. The developed method will be used to profile prenylquinones in various Arabidopsis mutants as well as in other commercially relevant crop species.

\section{Methods \\ Chemicals}

The solvents used for extraction were methanol $(\mathrm{MeOH}$, HPLC grade, Chromanorm), chloroform $\left(\mathrm{CHCl}_{3}\right.$, analytical grade, Normapur) and tetrahydrofuran (THF, analytical grade, Normapur) from VWR (Leuven, Belgium), isopropanol (IPA, HPLC grade) and ethylacetate (EtAc, analytical grade) from Acros Organics (Geel, Belgium). ULC/MS grade $\mathrm{MeOH}$ and water from Biosolve (Valkenswaard, The Netherlands) were used for the UHPLCAPCI-QTOFMS analyses.

$\alpha-\mathrm{T}$ and $\mathrm{K}$ standards of HPLC grade ( $\geq 99.5 \%$ ) were purchased from Sigma-Aldrich (Steinheim, Germany). Decyl-plastoquinone $(\sim 75 \%)$ was obtained from SigmaAldrich. PQ-9 and PC-8 standards were provided by Jerzy Kruk (Jagiellonian University, Kraków, Poland). The oxidized and reduced PQ-9 standards were obtained as described in [25] with slight changes. Briefly, an excess $(1 \mu \mathrm{g})$ of sodium borohydride (Fluka, Buchs, Switzerland) was added to the oxidized PQ standard (100 ng) to completely reduce it to $\mathrm{PQH}_{2}$. The retention time of both forms was then determined by UHPLCAPCI-QTOFMS. Ascorbic acid was purchased from Carl Roth (Karlsruhe, Germany) and butyl hydroxytoluene (BHT) from Sigma-Aldrich.

\section{Plant material and treatments}

Arabidopsis thaliana (Columbia-0) plants were grown on soil under standard growth conditions $\left(150 \mu \mathrm{E} \cdot \mathrm{m}^{-2} \cdot \mathrm{s}^{-}\right.$ ${ }^{1}, 8 / 16 \mathrm{~h}$ light/dark period, $21 / 18^{\circ} \mathrm{C}, 55 \%$ relative air humidity) according to the protocol described in [26] with slight modifications. HL treatment was performed on 4- to 5-week-old rosettes by exposure to continuous $\mathrm{HL}$ conditions $\left(500 \mu \mathrm{E} \cdot \mathrm{m}^{-2} \cdot \mathrm{s}^{-1}, 21^{\circ} \mathrm{C}, 55 \%\right.$ relative air humidity) for 1 week in a PGC 6HID growth chamber (Percival Scientific, Boone, IA) equipped with $400 \mathrm{~W}$ metal halide lamps (Philips).

\section{Prenyllipid extraction}

Arabidopsis leaves from 4- to 5-week-old rosettes were ground in a mortar with liquid nitrogen. Approximately $100 \mathrm{mg}$ of leaf material was then exactly weighed, transferred to a $1.5 \mathrm{~mL}$ microcentrifuge tube (Eppendorf, Hamburg, Germany) and swiftly re-suspended in five volumes of the selected solvent (e.g. $500 \mu \mathrm{L}$ for $100 \mathrm{mg}$ ) containing decylplastoquinone at $2 \mu \mathrm{g} / \mathrm{mL}$ as internal standard. Care was taken that no thawing occurred 
before the solvent was added. Glass beads of about 1 $\mathrm{mm}$ of diameter (Assistent, Sontheim, Germany) were added and samples were further homogenized for $3 \mathrm{~min}$ at $30 \mathrm{~Hz}$ in a tissue lyser (Retsch MM 300, Haan, Germany). Tubes were centrifuged on a benchtop centrifuge $\left(14,000 \times \mathrm{g}\right.$ for $3 \mathrm{~min}$ at $\left.4^{\circ} \mathrm{C}\right)$ and $400 \mu \mathrm{L}$ of supernatant was then transferred to an appropriate glass vial for immediate UHPLC-QTOFMS analysis.

\section{Liquid chromatography-mass spectrometry analysis}

The LC-MS system consisted of a Waters Acquity UPLC $^{\text {TM }}$ (Milford, MA) coupled to a Waters Synapt G2 MS QTOF equipped with an atmospheric pressure chemical ionization (APCI) source. Prenyllipids were separated on an Acquity BEH C18 column $(50 \times 2.1 \mathrm{~mm}$, $1.7 \mu \mathrm{m})$ under the following conditions: Solvent $\mathrm{A}=$ water; Solvent B $=\mathrm{MeOH}$; $90-100 \% \mathrm{~B}$ in $1.5 \mathrm{~min}, 100 \%$ $\mathrm{B}$ for $2.5 \mathrm{~min}$, re-equilibration at $90 \% \mathrm{~B}$ for $0.5 \mathrm{~min}$. The flow rate was $800 \mu \mathrm{L} / \mathrm{min}$ and the injection volume was $2.5 \mu \mathrm{L}$. The temperature of the column was set to $60^{\circ} \mathrm{C}$ and the autosampler chamber was kept at $15^{\circ} \mathrm{C}$. Data were acquired with a scan time of $0.4 \mathrm{~s}$ over an $\mathrm{m} /$ $z$ range of 225-1200 in the negative ion MS mode. The corona current was set to $18 \mu \mathrm{A}$ and the cone voltage to $40 \mathrm{~V}$. The source temperature was maintained at $120^{\circ} \mathrm{C}$ and the APCI probe temperature at $475^{\circ} \mathrm{C}$. The desolvation gas flow was set to $800 \mathrm{~L} / \mathrm{hr}$. The mobile phase was diverted to waste for $0.3 \mathrm{~min}$ at the beginning of the gradient. Accurate mass measurements were obtained by infusing a $400 \mathrm{ng} / \mathrm{mL}$ solution of the small peptide leucin-enkephalin at a flow rate of $10 \mu \mathrm{L} / \mathrm{min}$ through the Lock Spray ${ }^{\mathrm{TM}}$ probe. For the identification of prenyllipids, positive and negative ion MS/MS experiments were carried out using a fixed collision energy of $40 \mathrm{eV}$ and argon as collision gas at a flow of $2.1 \mathrm{~mL} /$ min. The quadrupole LM resolution was 4.7 , and the HM resolution was 15 . MS/MS product ion spectra were acquired over the $m / z$ range 50-1200. Absolute quantities of prenyllipids were determined using standard curves obtained from standard compounds. The concentrations of the calibration points for $\alpha-\mathrm{T}$ were $0.05,0.2,1.0,2.0$, and $10.0 \mu \mathrm{g} / \mathrm{mL}$, for PQ-9 and PC-8 $0.05,0.2,1.0,2.0$, and $5.0 \mu \mathrm{g} / \mathrm{mL}$. For $\mathrm{K}$, the concentrations were $0.1,0.25,1.0$, and $2.5 \mu \mathrm{g} / \mathrm{mL}$. All standard solutions contained decylplastoquinone (internal standard) at a concentration of $2 \mu \mathrm{g} / \mathrm{mL}$.

\section{Data treatment}

Data were processed using Masslynx v4.1 (Waters). Multivariate analysis was carried out using MarkerLynx $\mathrm{XS}^{\mathrm{TM}}$ (Waters). The following parameters were used: initial and final retention times 0.7-3.0 min, mass range $m / z$ 225-1200 Da, mass tolerance $0.03 \mathrm{Da}$, retention time window $0.10 \mathrm{~min}$, automatic peak width detection, intensity threshold 1000 counts. The deisotope filtering function was applied. Non-normalized peak areas were generated. Variables were UV-scaled before applying PCA.

\section{Acknowledgements}

We thank Jerzy Kruk for kindly providing purified plastoquinone-9 and plastochromanol-8 standards. GG acknowledges support from the Swiss Plant Science Web. FK was supported by UniNE, SystemsX PGCE, NCCR Plant Survival and SNF 31003A_127380.

\section{Author details}

${ }^{1}$ Laboratory of Plant Physiology, University of Neuchâtel, 2000 Neuchâtel, Switzerland. ${ }^{2}$ Chemical Analytical Service of the Swiss Plant Science Web, University of Neuchâtel, 2000 Neuchâtel, Switzerland.

\section{Authors' contributions}

JM grew, collected, and extracted plants. GG developed the UHPLC-APCIQTOFMS method and conducted measurements. JM and GG carried out the optimization of the sample preparation, performed data analysis and treatment. GG and JM wrote the manuscript. FK contributed to the writing of the manuscript. All authors conceived the study. All authors read, commented and approved the final manuscript.

\section{Competing interests}

The authors declare that they have no competing interests.

Received: 19 May 2011 Accepted: 21 July 2011 Published: 21 July 2011

\section{References}

1. Nowicka B, Kruk J: Occurrence, biosynthesis and function of isoprenoid quinones. BBA-Bioenergetics 2010, 1797:1587-1605.

2. Mene-Saffrane $L$, Jones AD, DellaPenna D: Plastochromanol-8 and tocopherols are essential lipid-soluble antioxidants during seed desiccation and quiescence in Arabidopsis. P Natl Acad Sci USA 2010, 107:17815-17820.

3. Kobayashi N, DellaPenna D: Tocopherol metabolism, oxidation and recycling under high light stress in Arabidopsis. Plant J 2008, 55:607-618.

4. Lichtenthaler HK: Biosynthesis, accumulation and emission of carotenoids, alpha-tocopherol, plastoquinone, and isoprene in leaves under high photosynthetic irradiance. Photosynth Res 2007, 92:163-179.

5. Krieger-Liszkay A, Trebst A: Tocopherol is the scavenger of singlet oxygen produced by the triplet states of chlorophyll in the PSII reaction centre. J Exp Bot 2006, 57:1677-1684.

6. Havaux M, Eymery F, Porfirova S, Rey P, Dormann P: Vitamin E protects against photoinhibition and photooxidative stress in Arabidopsis thaliana. Plant Cell 2005, 17:3451-3469.

7. Kruk J, Trebst A: Plastoquinol as a singlet oxygen scavenger in photosystem II. BBA-Bioenergetics 2008, 1777:154-162.

8. Szymanska R, Kruk J: Identification of hydroxy-plastochromanol in Arabidopsis leaves. Acta Biochim Pol 2010, 57:105-108.

9. Lohmann A, Schottler MA, Brehelin C, Kessler F, Bock R, Cahoon EB, Dormann P: Deficiency in phylloquinone (vitamin K-1) methylation affects prenyl quinone distribution, photosystem I abundance, and anthocyanin accumulation in the Arabidopsis AtmenG mutant. J Biol Chem 2006, 281:40461-40472.

10. Shimada H, Ohno R, Shibata M, Ikegami I, Onai K, Ohto M, Takamiya K: Inactivation and deficiency of core proteins of photosystems I and II caused by genetical phylloquinone and plastoquinone deficiency but retained lamellar structure in a T-DNA mutant of Arabidopsis. Plant $J$ 2005, 41:627-637.

11. Johnson TW, Shen GZ, Zybailov B, Kolling D, Reategui R, Beauparlant S, Vassiliev IR, Bryant DA, Jones AD, Golbeck JH, Chitnis PR: Recruitment of a foreign quinone into the $A(1)$ site of photosystem I - I. Genetic and physiological characterization of phylloquinone biosynthetic pathway mutants in Synechocystis sp PCC 6803. J Biol Chem 2000, 275:8523-8530.

12. Zbierzak AM, Kanwischer M, Wille C, Vidi PA, Giavalisco P, Lohmann A, Briesen I, Porfirova S, Brehelin C, Kessler F, Dormann P: Intersection of the 
tocopherol and plastoquinol metabolic pathways at the plastoglobule. Biochem J 2010, 425:389-399.

13. Kruk J, Karpinski S: An HPLC-based method of estimation of the total redox state of plastoquinone in chloroplasts, the size of the photochemically active plastoquinone-pool and its redox state in thylakoids of Arabidopsis. BBA-Bioenergetics 2006, 1757:1669-1675.

14. Yoshida K, Shibata M, Terashima I, Noguchi K: Simultaneous determination of in vivo plastoquinone and ubiquinone redox states by HPLC-based analysis. Plant Cell Physiol 2010, 51:836-841.

15. Byrdwell WC: Atmospheric pressure chemical ionization mass spectrometry for analysis of lipids. Lipids 2001, 36:327-346.

16. Swartz ME: UPLC (TM): An introduction and review. I Liq Chromatogr Relat Technol 2005, 28:1253-1263.

17. Nguyen DTT, Guillarme D, Rudaz S, Veuthey JL: Fast analysis in liquid chromatography using small particle size and high pressure. J Sep SCi 2006, 29:1836-1848.

18. Plumb R, Castro-Perez J, Granger J, Beattie I, Joncour K, Wright A: Ultraperformance liquid chromatography coupled to quadrupole-orthogonal time-of-flight mass spectrometry. Rapid Commun Mass Sp 2004, 18:2331-2337.

19. Lauridsen C, Leonard SW, Griffin DA, Liebler DC, McClure TD, Traber MG: Quantitative analysis by liquid chromatography tandem mass spectrometry of deuterium-labeled and unlabeled vitamin E in biological samples. Anal Biochem 2001, 289:89-95.

20. Lanina SA, Toledo P, Sampels S, Kamal-Eldin A, Jastrebova JA: Comparison of reversed-phase liquid chromatography-mass spectrometry with electrospray and atmospheric pressure chemical ionization for analysis of dietary tocopherols. J Chromatogr A 2007, 1157:159-170.

21. Kurilich AC, Britz SJ, Clevidence BA, Novotny JA: Isotopic labeling and LCAPCI-MS quantification for investigating absorption of carotenoids and phylloquinone from kale (Brassica oleracea). J Agric Food Chem 2003, 51:4877-4883.

22. Suhara $Y$, Kamao M, Tsugawa N, Okano T: Method for the determination of vitamin $\mathrm{K}$ homologues in human plasma using high-performance liquid chromatography-tandem mass spectrometry. Anal Chem 2005, 77:757-763.

23. Maeda H, Song W, Sage TL, DellaPenna D: Tocopherols play a crucial role in low-temperature adaptation and phloem loading in Arabidopsis. Plant Cell 2006, 18:2710-2732.

24. Szymanska R, Kruk J: Plastoquinol is the main prenyllipid synthesized during acclimation to high light conditions in Arabidopsis and is converted to plastochromanol by tocopherol cyclase. Plant Cell Physiol 2010, 51:537-545.

25. Kruk J: Charge-transfer complexes of plastoquinone and alphatocopherol quinone in vitro. Biophys Chem 1988, 30:143-149.

26. Hiltbrunner A, Bauer J, Alvarez-Huerta M, Kessler F: Protein translocon at the Arabidopsis outer chloroplast membrane. Biochem Cell Biol 2001, 79:629-635.

doi:10.1186/1746-4811-7-23

Cite this article as: Martinis et al: A novel method for prenylquinone profiling in plant tissues by ultra-high pressure liquid chromatographymass spectrometry. Plant Methods 2011 7:23.

\section{Submit your next manuscript to BioMed Central and take full advantage of:}

- Convenient online submission

- Thorough peer review

- No space constraints or color figure charges

- Immediate publication on acceptance

- Inclusion in PubMed, CAS, Scopus and Google Scholar

- Research which is freely available for redistribution

Submit your manuscript at www.biomedcentral.com/submit
Biomed Central 